\title{
Vault Hematoma after Vaginal Hysterectomy: An Invariable Complication
}

\author{
Laila Yahya A. Alhubaishi' ${ }^{1}$ Atif Bashir Fazari ${ }^{1,2^{*}}$ \\ ${ }^{1}$ Department of Obstetrics \& Gynecology, Latifa Hospital, DHA, Dubai, UAE \\ ${ }^{2}$ Faculty of Medicine, University of Medical Sciences \& Technology, Khartoum, Sudan \\ Email: *atiffazari@hotmail.co.uk
}

How to cite this paper: Alhubaishi, L.Y.A. and Fazari, A.B. (2019) Vault Hematoma after Vaginal Hysterectomy: An Invariable Complication. Open Journal of Obstetrics and Gynecology, 9, 578-581.

https://doi.org/10.4236/ojog.2019.95056

Received: April 4, 2019

Accepted: May 5, 2019

Published: May 8, 2019

Copyright () 2019 by author(s) and Scientific Research Publishing Inc. This work is licensed under the Creative Commons Attribution International License (CC BY 4.0).

http://creativecommons.org/licenses/by/4.0/

\section{cc) (7) Open Access}

\begin{abstract}
Hysterectomy is common major operation performed in gynecological field practice after Caesarean section. Vaginal hysterectomy is preferred surgery for uterovaginal prolapsed. Vaginal hysterectomy has advantages over abdominal hysterectomy. Post hysterectomy hematomas are responsible for serious morbidity. Ultrasound is an accurate diagnostic tool and so Computed tomography. Conservative management, analgesia and antibiotics will work in small hematoma, but the significant sized hematoma needs surgical drainage and the infected hematoma. Refinement in surgical techniques is recommended to minimize the formation vaginal vault hematomas after vaginal hysterectomy.
\end{abstract}

\section{Keywords}

Vault Hematoma, Vaginal Hysterectomy, Surgical Anatomy

Hysterectomy is the most common major operation performed in gynecological field practice after Caesarean section. It is well recognized that vaginal hysterectomy has advantages over abdominal hysterectomy. The incidence of vaginal hysterectomy is rising as it is increasingly done for non-prolapsed uterus.

With advanced improvement seen in surgical equipment and techniques, hysterectomies are increasingly performed with laparoscopy and related complications have decreased.

Several authors reported different surgical techniques for abdominal and vaginal hysterectomy including laparoscopic approach without substantial differences [1].

Vaginal hysterectomy is preferred surgery for uterovaginal prolapsed. Anterior colporrhaphy and posterior colpoperineorrhaphy can be conveniently per- 
formed. It has less febrile morbidity, less risk of hemorrhage, almost no blood transfusions, shorter hospitalization, and quick convalescence as compared to abdominal route.

Abdominal hysterectomy has its own indication identity like gynecological tumors, recurrence pelvic or abdominal surgery and whenever a well access surgical field is needed.

The selection of cases for open, laparoscopic or vaginal approach is based on the indication, experience and case circumstances during surgery.

Although most of women with vaginal hysterectomy experience an uneventful postoperative course, hysterectomy is associated with non-negligible risks. Potentially serious postoperative complications such as, hemorrhage, infections, urinary and bowel injuries, may be either recognized intraoperatively, manifest during postoperative hospitalization, or sometimes become apparent weeks or months later like urinary incontinence and later vault prolapse.

One of the complications associated with vaginal hysterectomy is the formation of an infected pelvic hematoma manifesting as abdominal pain, fever and a foul-smelling discharge. These hematomas can be diagnosed by sonography and require treatment ranging from pain control and antibiotics to surgical drainage.

Post hysterectomy hematomas are responsible for serious morbidity especially if they are large and infected. The incidence of pelvic hematomas in general (infected and non-infected) varies between $25 \%$ and $98 \%$. The incidence of infected hematomas is estimated to be around 6\% - 9\% [2].

Rarely there can be collection of lymph, serous fluid or necrotic debris at different sites after hysterectomy. Mostly the collection occurred in the dependent areas. Hematomas can be formed in pouch of Douglas, subvesical space, ischio-rectal fossa and broad ligament [3].

It is difficult to diagnose hematoma by routine clinical examination only. Many patients may be asymptomatic; where as some may present with postoperative bleeding per vaginum (spotting to profuse bleeding per vaginum), postoperative discomfort, abdominal distension, paralytic ileus, continuous fever, foul smelling discharge per vaginum, abscess formation, tenesmus, nausea, vomiting, and diarrhea [4]. Post-operative pyrexia may be the hall presentation of hematoma after vaginal hysterectomy in such presentation exclusion of hematoma is articulate step in case management.

Ultrasound, being an accessible, non-invasive and accurate diagnostic tool, seems to be the modality of choice for diagnosing postoperative vault hematoma [5]. In a study, the overall incidence of vault hematoma was $19.4 \%, 70 \%$ had small-sized hematoma and 30\% had large sized hematoma [1].

Small vaginal vault hematomas $(2-3.9 \mathrm{~cm})$ unlikely to cause postoperative morbidity and can be managed expectantly. They can be left alone with watchful expectancy and follow-up ultrasonography for resolution of the hematoma done weekly [4].

Moderate $(4-5.9 \mathrm{~cm})$ and large $(>6 \mathrm{~cm})$ hematomas need further manage- 
ment. An extended morbid and complicated postoperative course can be alleviated if the hematoma can be drained. A small drain may be inserted through the drainage tract and left in place for a day or so. If the hematoma can be drained the patient's recovery will be more prompt.

When hematoma is clinically suspected confirmation will be by Ultrasound scan (USS) in addition multidetector Computed tomography (CT) represents the ideal modality to visualize the surgically altered pelvicanatomy and to consistently triage the varied spectrum of possible injuries. Serial inflammatory, septic markers, and culture studies from vault are crucial for diagnosis of any infected hematoma well needed in follow up for improvement.

The intervention includes the symptomatic relieve of pain and febrile morbidity with good analgesia and antibiotics coverage till further sensitivity results. Drainage of the hematoma is the ideal option of treatment when it is big ( $>4$ $\mathrm{cm})$, infected one or does not respond to conservative choice.

Presence of hematoma even without infection at the site vault interferes with healing process and weakens the wound integrity leads to dehiscence of the wound at early post-operatively and later results in weak scar and vault prolapse

A significant reduction in the incidence of infected pelvic hematoma following vaginal hysterectomy was noted using a surgical technique that allows for drainage of blood and debris through the vaginal cuff. Most properly because of good skills, well orientation of clinical applied anatomy during the training and improved training.

Clinical orientation of the surgical anatomy during the surgical techniques and expert skill is important along of performance of surgery under direct supervision with safe hemostasis play role in less hematoma formation. Good case selection for vaginal approach for hysterectomy is important. Early detection and prompt intervention will improve the outcome of this morbidity.

Refinement in surgical techniques is recommended to minimize the risk of clinically significant vaginal vault hematomas after vaginal hysterectomy [6].

\section{Funding}

No funding sources.

\section{Ethical Approval}

Not required.

\section{Conflicts of Interest}

The authors declare no conflicts of interest regarding the publication of this paper.

\section{References}

[1] Iaco, P.D., Ceccaroni, M., Alboni, C., Roset, B., Sansovini, M., D’Alessandro, L., et al. (2006) Transvaginal Evisceration after Hysterectomy: Is Vaginal Cuff Closure 
Associated with a Reduced Risk? European Journal of Obstetrics \& Gynecology and Reproductive Biology, 125, 134-138. https://doi.org/10.1016/j.ejogrb.2005.08.009

[2] Geeta, D., Ravikanth, G. and Tanushree, R. (2014) Vaginal Removal of Non-Prolapsed Uterus with Benign Pathology. Indian Medical Gazette, 123-128.

[3] Chaudhry, S.M. and Anwer, S. (2016) Presentation and Management of Vaginal Vault Hematoma after Hysterectomy. Journal of Surgery Pakistan, 21, 35-38. https://doi.org/10.21699/jsp.21.1.9

[4] Dane, C., Dane, B., Cetin, A. and Yayla, M. (2007) Sonographically Diagnosed Vault Hematomas Following Vaginal Hysterectomy and Its Correlation with Postoperative Morbidity. Infectious Diseases in Obstetrics and Gynecology, 2007, Article ID: 91708. https://doi.org/10.1155/2007/91708

[5] Kho, R.M., Hilger, W.S., Hentz, J.G., Magtibay, P.M. and Magrina, J.F. (2007) Robotic Hysterectomy: Technique and Initial Outcomes. American Journal of Obstetrics \& Gynecology, 197, 113.e1-113.e4. https://doi.org/10.1016/j.ajog.2007.05.005

[6] Spencer, C., Fiadjoe, P. and Abdel Razzek, A. (2012) Role of Routine Vault Drainage at Vaginal Hysterectomy. BJOG, 119, 1015-1016.

https://doi.org/10.1111/j.1471-0528.2012.03316.x 